\title{
Adipocyte-specific deletion of $m$ TOR inhibits adipose tissue development and causes insulin resistance in mice
}

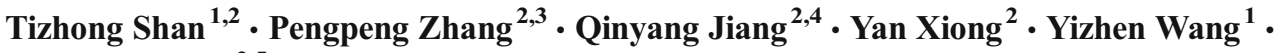 \\ Shihuan Kuang ${ }^{2,5}$
}

Received: 22 December 2015 / Accepted: 20 May 2016/Published online: 13 June 2016

(C) Springer-Verlag Berlin Heidelberg 2016

\begin{abstract}
Aims/hypothesis The in vivo role of mechanistic target of rapamycin (mTOR) in the development and function of adipose tissue, especially brown adipose tissue (BAT), is not well understood. Here, we aimed to assess the effect of $m$ TOR (also known as Mtor) knockout on adipose tissues and systemic energy metabolism.

Methods We generated adipocyte-specific mTOR-knockout mice (Adipoq-mTOR) by crossing adiponectin-Cre (Adipoq-Cre) mice with $m T O R^{\text {flox/flox }}$ mice. The mice were then subjected to morphological, physiological (indirect calorimetry, glucose and insulin tolerance tests)
\end{abstract}

Tizhong Shan, Pengpeng Zhang, Qinyang Jiang and Yan Xiong contributed equally to this paper.

Electronic supplementary material The online version of this article (doi:10.1007/s00125-016-4006-4) contains peer-reviewed but unedited supplementary material, which is available to authorised users.

Tizhong Shan

tzshan@zju.edu.cn

Shihuan Kuang

skuang@purdue.edu

1 College of Animal Sciences, Zhejiang University, 866 Yuhangtang Road, Hangzhou 310058, People's Republic of China

2 Department of Animal Sciences, Purdue University, 901 West State Street, West Lafayette, IN 47907, USA

3 College of Life Sciences, Xinyang Normal University, Xinyang, People's Republic of China

4 College of animal science and technology, Guangxi University, Nanning, People's Republic of China

5 Purdue University Center for Cancer Research, West Lafayette, IN 47907, USA and gene expression analyses to determine the role of mTOR in adipose tissues.

Results We provide in vivo evidence that $m T O R$ is essential for adipose tissue development and growth. Deletion of $m T O R$ decreased the mass of both BAT and white adipose tissues (WAT) and induced browning of WAT. In addition, ablation of $m T O R$ in adipose tissues caused insulin resistance and fatty liver in the Adipoq-mTOR mice. Furthermore, $m$ TOR was required for adipocyte differentiation in vivo and activation of PPAR $\gamma$ ameliorated the differentiation deficiency of the $m T O R$-null adipocytes.

Conclusions/interpretation Our findings demonstrate that mTOR is a critical regulator of adipogenesis and systemic energy metabolism. Our study provides key insights into the role of mTOR in adipose tissues; such knowledge may facilitate the development of novel strategies with which to treat obesity and related metabolic diseases.

Keywords Adipose $\cdot$ Browning $\cdot$ Insulin resistance $\cdot$ mTOR . PPAR $\gamma$

$\begin{array}{ll}\text { Abbreviations } \\ \text { asWAT } & \text { Anterior subcutaneous WAT } \\ \text { BAT } & \text { Brown adipose tissue } \\ \text { C/EBP } \alpha & \text { CCAAT/enhancer binding protein } \alpha \\ \text { eWAT } & \text { Epididymal WAT } \\ \text { FABP4 } & \text { Fatty acid binding protein } 4 \\ \text { H\&E } & \text { Haematoxylin and eosin } \\ \text { HFD } & \text { High-fat diet } \\ \text { ITT } & \text { Insulin tolerance test } \\ \text { iWAT } & \text { Inguinal WAT } \\ \text { KO } & \text { Knockout } \\ \text { mTOR } & \text { Mechanistic target of rapamycin } \\ \text { mTORC } & \text { mTOR complex }\end{array}$




$\begin{array}{ll}\text { PPAR } \gamma & \text { Peroxisome proliferator-activated receptor } \gamma \\ \text { RER } & \text { Respiratory exchange ratio } \\ \text { S6K1 } & \text { Ribosomal S6 kinase 1 } \\ \text { SVF } & \text { Stromal vascular fraction } \\ \text { TG } & \text { Triacylglycerol } \\ \text { UCP1 } & \text { Uncoupling protein 1 } \\ \text { WAT } & \text { White adipose tissue } \\ \text { WT } & \text { Wild-type }\end{array}$

\section{Introduction}

Adipose tissue plays a critical role in regulating energy balance and metabolism and its dysfunction is closely associated with metabolic diseases such as obesity and insulin resistance. In mammals, there are two main types of adipose: white adipose tissue (WAT) and brown adipose tissue (BAT). WAT is the major energy storage site that stores excess energy, whereas BAT contains numerous mitochondria with high levels of uncoupling protein 1 (UCP1) that dissipates energy as heat [1]. Adipocytes in the WAT and BAT are called white and brown adipocytes, respectively. Recently, a third type of adipocyte, called beige or brite [2], has been found in WAT. Beige adipocytes can be induced by chemical or hormonal stimulation, cold exposure or genetic manipulation [3-7]. Similar to classical brown adipocytes, beige adipocytes have high levels of UCP1 $[3,7,8]$. Both brown and beige adipocytes can increase energy expenditure and counteract obesity [7-9]. Thus, understanding the development and function of brown and beige adipocytes may provide novel strategies with which to counteract obesity.

Mammalian target of rapamycin (mTOR) is a conserved serine/threonine protein kinase involved in regulating protein synthesis, cell growth and energy metabolism [10]. mTOR functions in two distinct complexes: mTOR complex 1 (mTORC1) and mTORC2 [11]. mTORC1, consists of mTOR, raptor and deptor, is sensitive to rapamycin [10]. mTORC2, containing mTOR, rictor and deptor, is insensitive to rapamycin [10]. Recent studies demonstrate that both complexes play a crucial role in adipogenesis [12, 13], lipid homeostasis $[10,14,15]$, glucose metabolism and insulin actions $[16,17]$. Inhibition of mTORC1 signalling by rapamycin or small interfering RNA against Raptor (also known as Rptor) decreases adipocyte proliferation and differentiation [18-21] and protects mice against high-fat diet (HFD)-induced obesity [22]. Adipose-specific ablation of Raptor also enhances energy expenditure and protects the mutant mice against HFDinduced obesity [23, 24]. Mice lacking ribosomal S6 kinase 1 (S6K1), a key downstream target of mTORC1, have relatively lower fat mass and higher energy expenditure [13, 25]. Moreover, deletion or overexpression of $T s c 1 / T s c 2$, upstream regulators of mTORC1, further confirms a key role for mTORC1 in adipocyte differentiation and energy metabolism
[26]. Compared with mTORC1, the function of mTORC2 in adipocytes is not well understood. Rictor-null mice die at around embryonic day 10.5 [27, 28]. In Caenorhabditis elegans, Rictor-null worms have comparatively more body fat $[14,29]$, indicating that mTORC2 may also play a role in regulating adipogenesis. In mice, adipose-specific deletion of Rictor decreases glucose uptake and glucose tolerance in vivo without affecting adipocyte size or fat mass $[16,17,30]$. These reports indicate that $\mathrm{mTORC} 1$ and $\mathrm{mTORC} 2$ play distinct roles in adipogenesis and lipid metabolism. However, most of the earlier studies involved perturbations of either mTORC1 or mTORC1. The direct effect of genetic deletion of $m$ TOR (also known as Mtor), which has the effect of simultaneously suppressing mTORC1 and $\mathrm{mTORC} 2$, in adipose tissues has not been reported. Moreover, previous studies used FABP4-Cre to drive the adipose-specific deletion of Raptor or Rictor and this may have led to off-target deletion in nonadipose tissues $[31,32]$ and confound the interpretation of results. In this study, we directly and specifically deleted $m T O R$ in adipocytes by crossing adipocyte-restricted adiponectin-Cre (Adipoq-Cre) mice with $m T O R^{\text {flox/flox }}$ mice.

\section{Methods}

Animals All procedures involving mice were guided by Purdue University Animal Care and Use Committee. Additionally, all experimental protocols were approved by Purdue University Animal Care and Use Committee. Adipoq-Cre (stock no. 010803) and $m T O R^{\text {flox/flox }}$ (stock no. 011009) mice were purchased from Jackson Laboratory (Bar Harbor, ME, USA). Mice were of a C57BL/6 J background and housed in an animal facility with free access to water and standard rodent chow food or HFD (TD.06414; Harlan, Indianapolis, IN, USA). PCR genotyping (Sigma-Aldrich, St Louis, MO, USA) was carried out as described by the supplier.

Indirect calorimetry study Oxygen consumption $\left(\dot{V} \mathrm{O}_{2}\right)$, carbon dioxide production $\left(\dot{V} \mathrm{CO}_{2}\right)$, respiratory exchange ratio (RER) and heat production were measured by using an indirect calorimetry system (Oxymax, Columbus Instruments, Columbus, OH, USA) [7].

GTTs and insulin tolerance tests GTTs and insulin tolerance tests (ITT) were performed as described previously [7].

Haematoxylin-eosin staining For adipose tissues, section and haematoxylin and eosin (H\&E) staining was carried out as described previously [7]. For liver tissues, frozen livers were cut into $10 \mu \mathrm{m}$ thick cross sections using a Leica CM1850 cryostat (Leica Microsystems, Wetzlar, Germany) for H\&E staining. Whole-slide digital images were collected at magnification $\times 20$ with an Aperio Scan Scope slide scanner 
(Aperio, Vista, CA, USA). Scanned images were analysed by Photoshop CS3 (San Jose, CA, USA) to calculate nuclei numbers.

Primary adipocyte isolation and culture The BAT and WAT stromal vascular fraction (SVF) cells were isolated using collagenase digestion [7]. For adipogenic differentiation, cells were induced to differentiate when they reached $90 \%$ confluence [7].

Oil Red O staining Cultured cells or liver sections were stained using Oil Red O work solutions containing $6 \mathrm{ml}$ stock solution ( $5 \mathrm{~g} / \mathrm{l}$ in isopropanol) and $4 \mathrm{ml}$ double-distilled $\mathrm{H}_{2} \mathrm{O}$ for $30 \mathrm{~min}$. After staining, the cells or liver sections were washed and pictured.

Triacylglycerol and NEFA assay The triacylglycerol (TG) levels in liver, muscle and serum were determined using enzymatic methods (Cayman kit, no. 10010303; Ann Arbor, MI, USA). The levels of NEFA in serum were measured by fluorimetric assay kit (Cayman, no. 700310).

Total RNA extraction, cDNA synthesis and real-time PCR Total RNA was extracted from cells or tissues using Trizol Reagent (Sigma-Aldrich). The purity and concentration of total RNA were measured and then $5 \mu \mathrm{g}$ of total RNA were reversed transcribed. Real-time PCR was carried out and the $2^{-\Delta \Delta C_{t}}$ method was used to analyse the relative changes in gene expression normalised against 18S rRNA as internal control [7].

Protein extraction and western blot analysis Total protein was extracted from cells or tissues using RIPA buffer [7]. The UCP1 antibody and the mitochondrial antibody cocktail were from Abcam (Abcam, Cambridge, MA, USA); the p-S6, S6, p-4EBP1, 4EBP1, p-AKT, AKT antibodies were from Cell Signalling Technology (Danvers, MA, USA); all other antibodies were from Santa Cruz Biotechnology (Santa Cruz Biotechnology, Dallas, TX, USA). Protein separation and western blot analysis were conducted as described previously [33].

Data analysis No data were excluded and all experimental data are presented as means \pm SEM. Comparisons were made by unpaired two-tailed Student's $t$ tests. Effects were considered significant at $p<0.05$. Samples were randomised. Experimenters were not completely blinded to group assignment and outcome assessment.

\section{Results}

Generation of adipocyte-specific $\boldsymbol{m}$ TOR-knockout mice To directly investigate the role of mTOR in adipogenesis, we used the Cre-loxP recombination system. $m T O R^{\text {flox/flox }}$ mice were crossed with Adipoq-Cre mice that express Cre recombinase under control of the adiponectin gene promoter. The adipocyte-specific expression pattern of Adipoq-Cre has been verified by previous studies [31, 34, 35]. Thus, in the Adipoq-Cre/mTOR flox/flox mice (abbreviated as Adipoq-mTOR) the exons 1-5 of $m$ TOR should be deleted in adipocytes, leading to loss of expression of mTOR protein (Fig. 1a). Real-time PCR and western blotting analysis confirmed the efficient and specific deletion of $m T O R$ in BAT as well as in inguinal WAT (iWAT), anterior subcutaneous WAT (asWAT) and epididymal WAT (eWAT) (Fig. 1b and electronic supplementary material [ESM] Fig. 1a, b) but not in non-adipose tissues including muscle, kidney, liver, heart and lung (Fig. 1c and ESM Fig. 1c, d).

Adipocyte-specific deletion of $\boldsymbol{m} T O R$ causes reduction of fat mass Adipoq-mTOR mice were born at expected Mendelian ratios and were indistinguishable from their wildtype (WT) littermates. On the normal chow diet, the Adipoq-mTOR mice showed a similar growth curve and food intake to the WT littermates (data not shown) and the body weight was similar for WT and knockout (KO) mice at 8 weeks of age (Fig. 1d). Notably, the adipose tissues from Adipoq-mTOR mice were smaller in size than those from WT mice (Fig. 1e). The mass of BAT, iWAT, asWAT and eWAT taken from Adipoq-mTOR mice was $55 \%, 69 \%, 86 \%$ and $43 \%$ lighter, respectively, than the mass of tissue taken from their WT littermates (Fig. 1f). The muscle mass in Adipoq-mTOR mice was unchanged (Fig. 1g). Strikingly, the liver, spleen and heart of the Adipoq-mTOR mice were 58\%, $42 \%$ and $32 \%$ heavier, respectively, than those of WT mice (Fig. 1g). The lipid and TG content was much higher in liver (ESM Fig. 2a-c) and skeletal muscles (ESM Fig. 2d) of Adipoq-mTOR mice compared with WT mice. In addition, we found lower TG levels and higher NEFA levels in serum of Adipoq-mTOR mice (ESM Fig. 2e, f). Taken together, adipocyte-specific deletion of $m T O R$ caused reduction of adipose tissues but expansion of non-adipose tissues, especially the liver.

Ablation of $\boldsymbol{m}$ TOR affects adipogenesis of brown adipocytes We next examined whether the decreased BAT mass in Adipoq-mTOR mice is due to reduction in adipocyte cell number, cell size or both. Results from H\&E staining and nuclei density analysis (number of nuclei per unit area) showed there to be a higher density of nuclei in the KO mice ( $335 \pm 21$ /area) than in the WT mice $(171 \pm 14$ /area), suggesting smaller BAT adipocyte size in the $\mathrm{KO}$ mice (Fig. 2a). Consistently, the genomic DNA content per BAT depot was lower in the Adipoq-mTOR mice than in the WT mice (Fig. 2b). Taken 
Fig. 1 Adipoq-Cre mediated deletion of $m$ TOR decreases fat mass. (a) Targeting strategy for deletion of $m T O R$. $(\mathbf{b}, \mathbf{c})$ mRNA levels of $m T O R$ in adipose $(\mathbf{b}, n=4)$ and non-adipose tissues (c, $n=3$ ). (d) The body weight of WT $\left(m T O R^{\text {flox/flox }}\right)$ and KO (Adipoq-mTOR) mice $(n=6)$. (e) Representative images of BAT and WAT depots. Scale bar, $3 \mathrm{~mm}$. (f, $\mathbf{g})$ The weights of adipose (f) and non-adipose tissues $(\mathbf{g})(n=6)$. Mice 8-10 weeks of age were used. White bars, WT mice; black bars, KO mice. Error bars represent SEM; ${ }^{*} p<0.05,{ }^{* *} p<0.01$ and $* * * p<0.001$ for KO vs WT. EDL, extensor digitorum longus muscle; Gas, gastrocnemius muscle; Hrt, heart; Kid, kidney; Liv, liver; Sol, soleus muscle; Spl, spleen; TA, tibialis anterior muscle
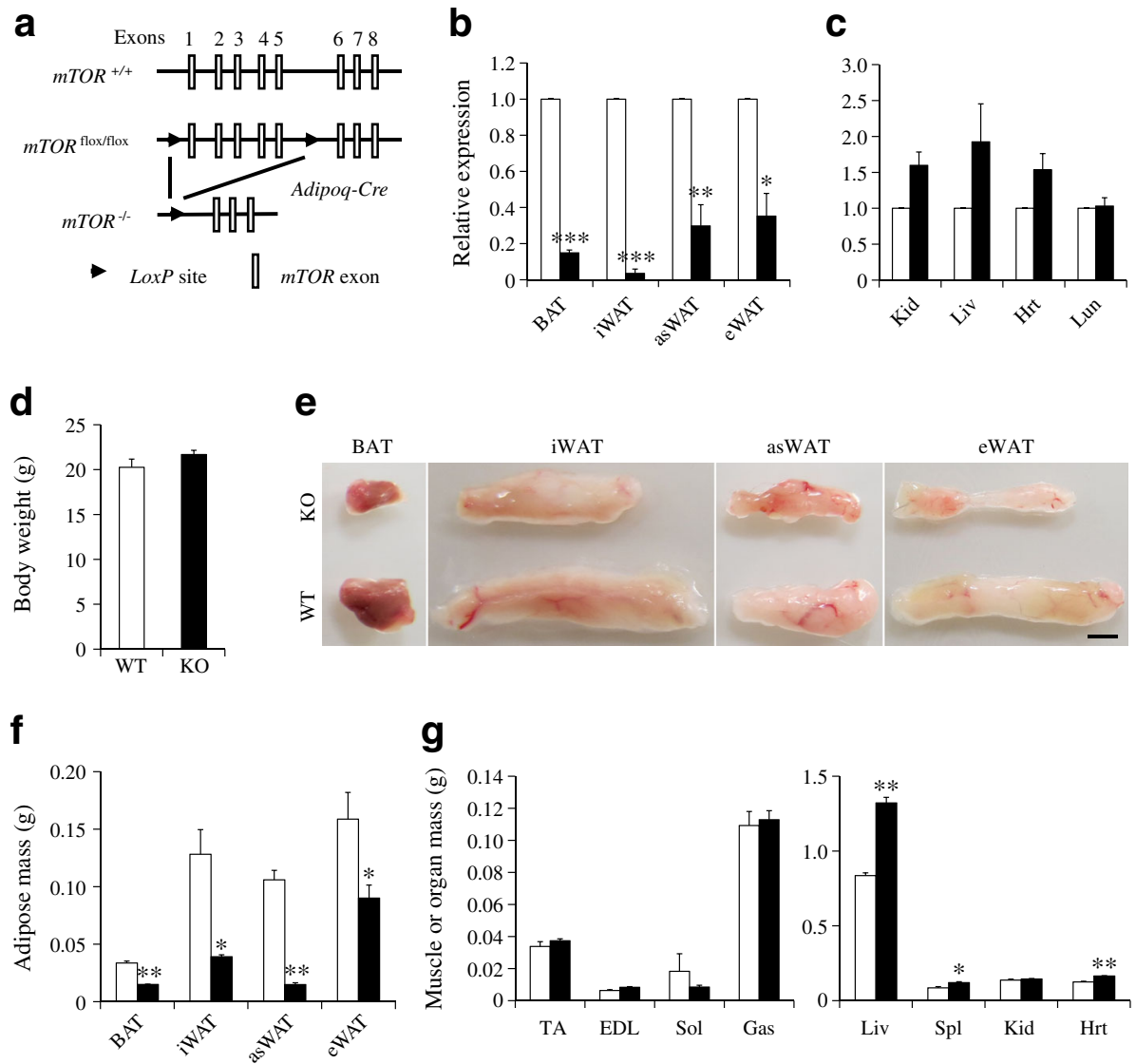

together, the reduced BAT mass in Adipoq-mTOR mice is a consequence of a decrease in cell size as well as cell numbers.

We next analysed the molecular phenotype of BAT from WT and Adipoq-mTOR mice. Mitochondrial biogenesis and brown adipocyte marker proteins and their genes, such as Ucp 1, Prdm16, Cidea and Ppara, were expressed at similar levels in WT and Adipoq-mTOR mice (Fig. 2c, d). However, the BAT from Adipoq-mTOR mice contained lower levels of fatty acid binding protein 4 (FABP4), a mature adipocyte marker (Fig. 2c). Consistently, the mRNA levels of mature adipocyte markers (Fabp4, Adipoq, Lep) were lower in the BAT of Adipoq-mTOR mice compared with WT mice (Fig. 2d). The expression levels of lipolysis-related genes Pnpla 2 and Lipe were unchanged (Fig. 2e) by $m$ TOR deletion. These data strongly suggest that deletion of $m T O R$ inhibits brown adipocyte adipogenesis in vivo.

To verify whether deletion of $m T O R$ affects brown preadipocyte differentiation in culture, we isolated BAT SVF cells from the WT and Adipoq-mTOR mice. We observed that $m$ TOR depletion had a more dramatic effect on BAT in vitro than in vivo (ESM Fig. 3a). mTOR deletion inhibited brown adipocyte differentiation and TG accumulation (ESM Fig. 3a). Consistent with the in vivo data, $m$ TOR-deficient brown adipocytes expressed lower levels of FABP4 and Adipoq in culture (ESM Fig. 3b, c). In addition, the expression of Ucpl and Cidea was also decreased by $m$ TOR knockout (ESM Fig. 3c). Together, these results indicate that deletion of $m T O R$ inhibits brown adipocyte differentiation and adipogenesis in vivo and in vitro.

Deletion of $\boldsymbol{m}$ TOR induces browning of iWAT To investigate the effect of $m T O R$ deficiency on WAT, we examined the morphology of iWAT. Interestingly, H\&E staining revealed that the iWAT of Adipoq-mTOR mice contained numerous brown adipocyte-like cells filled with multilocular lipid droplets (Fig. 3a). The iWAT of KO mice had a higher number of nuclei per area than iWAT of WT mice (WT 24 \pm 3 vs KO $110 \pm$ 11) but had a similar genomic DNA content per depot (Fig. 3a, b). We also observed more robust integrin $\alpha \mathrm{M}$ (Cd11b) labelling in the iWAT of KO compared with WT mice (data not shown), indicative of increased inflammatory infiltration. In addition, deletion of $m T O R$ decreased the expression of FABP4 (Fig. 3c). Interestingly, higher levels of UCP1 protein were detected in the iWAT of Adipoq-mTOR mice compared with WT mice (Fig. 3c). Consistently, Adipoq-mTOR iWAT expressed higher levels of BAT signature markers Ucp1, Cidea and Ppara (Fig. 3d). Also, mTOR deletion upregulated the expression of Lipe but downregulated the expression of Adipoq, Lep and Pnpla2 in iWAT (Fig. 3d, e). These data suggest that lack of $m T O R$ inhibits the development of iWAT but induces its browning. 
a

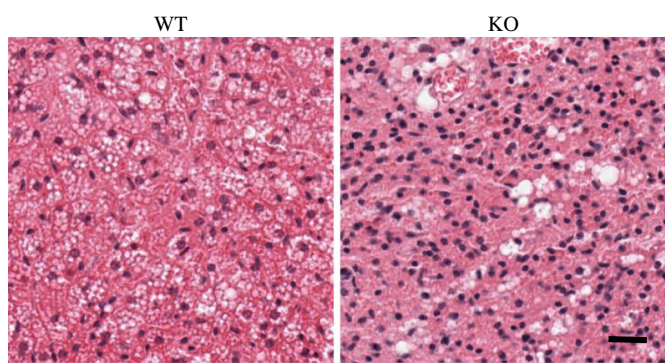

b

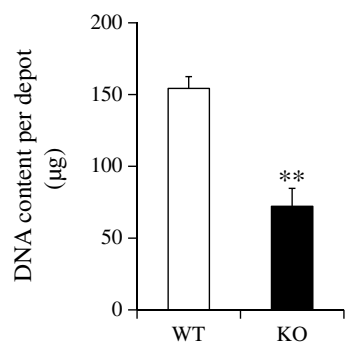

C

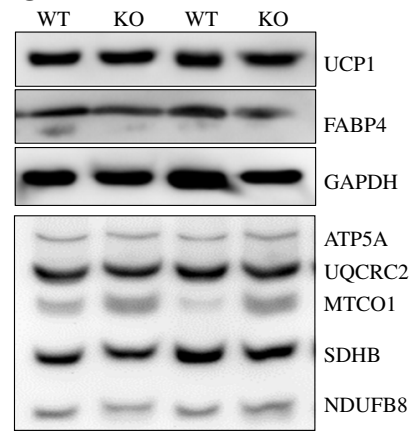

d

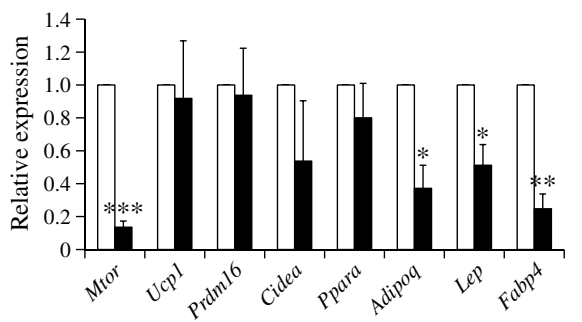

e

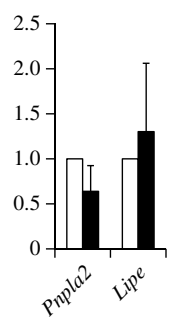

Fig. 2 mTOR deficiency inhibits BAT development. (a) H\&E staining of BAT sections. Scale bar, $100 \mu \mathrm{m}$. (b) Genomic DNA content per BAT depot $(n=4)$. (c) Levels of UCP1, FABP4 and mitochondrial proteins in BAT. (d, e) mRNA levels of mature adipocyte marker genes and BATselective genes $(\mathbf{d}, n=4$ or 5$)$ and lipolysis-related genes $(\mathbf{e}, n=3)$ in BAT from WT and KO mice. White bars, WT mice; black bars, KO mice. Error bars represent SEM; ${ }^{*} p<0.05,{ }^{* *} p<0.01$ and ${ }^{* * *} p<0.001$ for KO vs

To examine whether the effects of $m T O R$ deletion on browning of iWAT is cell autonomous, we isolated SVF cells from iWAT of WT and Adipoq-mTOR mice. Oil Red O staining
WT. ATP5A, ATP synthase, $\mathrm{H}^{+}$transporting, mitochondrial F1 complex, alpha subunit 1; GAPDH, glyceraldehyde-3-phosphate; dehydrogenase; MTCO1, mitochondrially encoded cytochrome c oxidase I; NDUFB8, NADH dehydrogenase (ubiquinone) 1 beta subcomplex 8; SDHB, succinate dehydrogenase complex, subunit B, iron sulfur (Ip); UQCRC2, ubiquinol cytochrome $\mathrm{c}$ reductase core protein 2

indicated that deletion of $m T O R$ inhibits adipocyte differentiation and TG accumulation (ESM Fig. 4a). Notably, the expression level of Ucpl and mature adipocyte markers was
Fig. 3 mTOR deficiency inhibits WAT development and induces browning of WAT. (a) H\&E staining of iWAT sections. Scale bar, $100 \mu \mathrm{m}$. (b) Genomic DNA content per iWAT depot of WT and KO mice $(n=4)$. (c) Protein levels of UCP1 and FABP4 in iWAT. (d, e) mRNA levels of mature adipocyte marker genes and BAT-selective genes (d) and lipolysis-related genes (e) in iWAT from WT and KO mice $(n=5)$. White bars, WT mice; black bars, $\mathrm{KO}$ mice. Error bars represent SEM; $* p<0.05$, $* * p<0.01$ and $* * * p<0.001$ for KO vs WT. GAPDH, glyceraldehyde 3-phosphate dehydrogenase a

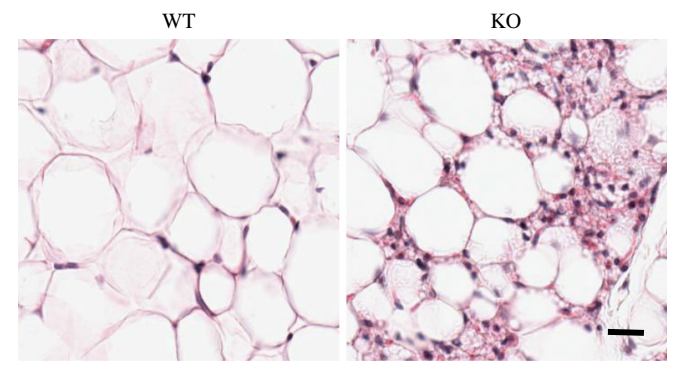

b

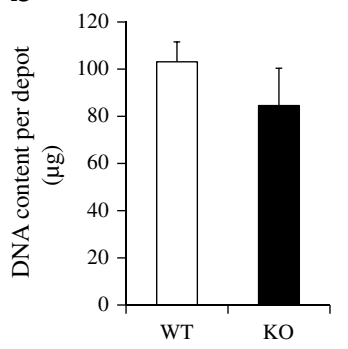

d

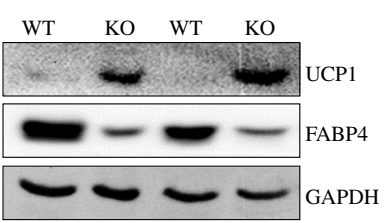

C

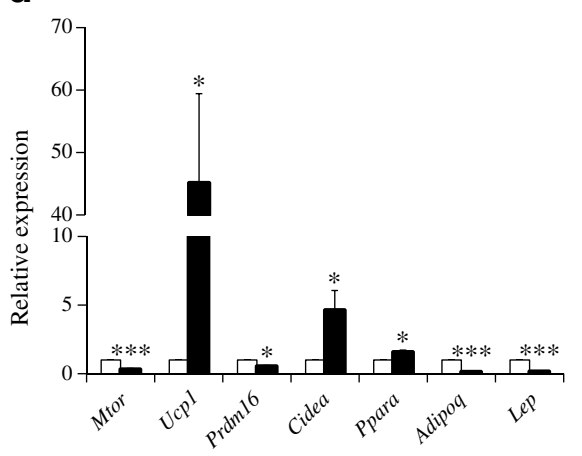

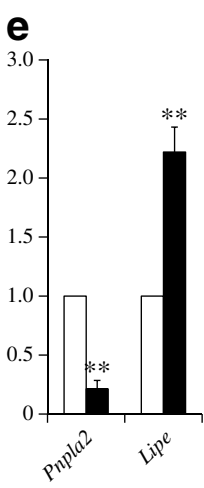


significantly lower in $m T O R$ KO white adipocytes (ESM Fig. $4 \mathrm{~b}, \mathrm{c})$. These results indicated that deletion of $m T O R$ induces browning of WAT in a non-cell autonomous manner.

$m T O R$ deletion affects insulin sensitivity but not energy metabolism Adipose tissues regulate systemic glucose metabolism and insulin sensitivity [7, 36]. To determine whether reduction of BAT and WAT mass in Adipoq-mTOR mice affects systemic glucose metabolism and insulin sensitivity, we conducted GTTs and ITTs. Compared with WT littermates, Adipoq-mTOR mice had higher blood glucose levels after glucose injection (Fig. 4a) and a slower rate of insulin-stimulated glucose clearance (Fig. 4b). However, compared with WT mice, the Adipoq-mTOR mice had similar values for $\dot{V} \mathrm{O}_{2}, \dot{V} \mathrm{CO}_{2}$, heat production and RER (Fig. 4c-f). Taken together, these results suggest that adipocyte-specific deletion of $m$ TOR does not affect energy metabolism but results in insulin resistance.

To further confirm the effects of $m T O R$ deficiency on energy metabolism and insulin sensitivity, we fed the WT and KO mice with HFD. Although the body weight of Adipoq-mTOR mice and WT mice was similar before and after HFD feeding, the fat mass of the KO mice was consistently less than that of the WT mice (ESM Fig. 5a, b). The liver mass in the Adipoq-mTOR mice was dramatically increased, whereas other tissues, including kidney, heart, lung, tibialis anterior muscle and gastrocnemius muscle, were unchanged (ESM Fig. 5c, d). Notably, the Adipoq-mTOR mice retained insulin resistance after HFD feeding (ESM Fig. 5e, f). These results together suggest that adipocyte-specific deletion of $m T O R$ causes insulin resistance regardless of dietary conditions.
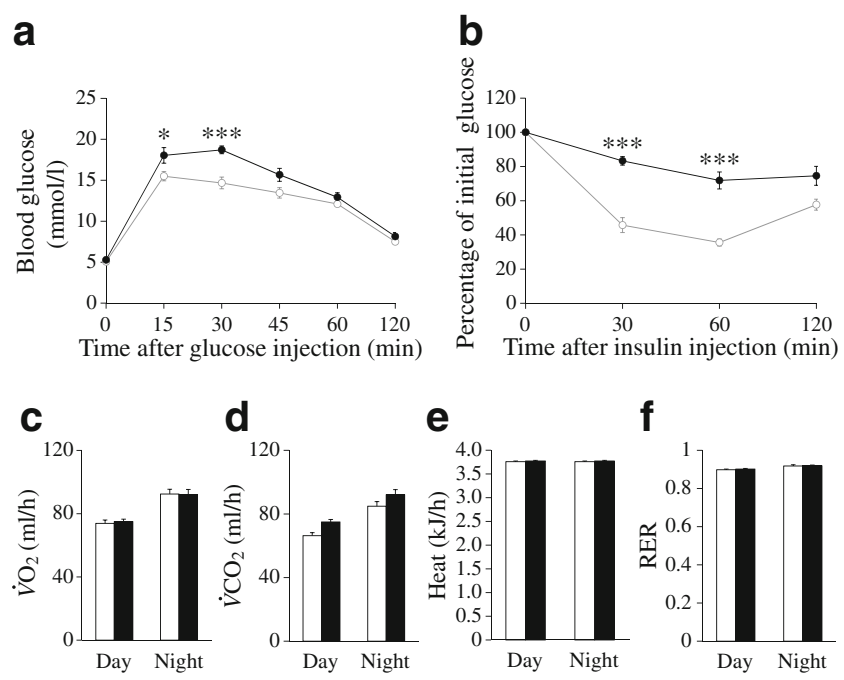

Fig. 4 Induced insulin resistance in Adioq-mTOR mice. (a, b) Blood glucose concentrations during IPGTT $(\mathbf{a}, n=8)$ and ITT $(\mathbf{b}, n=7)$ performed on WT and KO mice. (c-f) Average oxygen consumption (c), $\mathrm{CO}_{2}$ production (d), heat production (e) and RER (f) of WT and KO mice $(n=6)$. White circles and bars, WT mice; black circles and bars, $\mathrm{KO}$ mice. Error bars represent SEM; ${ }^{*} p<0.05$ and $* * * p<0.001$ for KO vs WT $m$ TOR affects adipogenesis through PPAR $\gamma$ To understand the molecular mechanism through which $m T O R$ affects fat development and adipogenesis, we examined peroxisome proliferator-activated receptor $\gamma(\operatorname{PPAR} \gamma)$ and CCAAT/ enhancer binding protein $\alpha(\mathrm{C} / \mathrm{EBP} \alpha)$, two key transcription factors that regulate adipogenesis [37]. Western blotting and real-time PCR results showed that $m T O R$ deficiency dramatically decreased the expression of PPAR $\gamma$ and $\mathrm{C} / \mathrm{EBP} \alpha$ in BAT (Fig. 5a, b), as well as in brown adipocytes (Fig. 5c). Similar results were obtained using iWAT tissue and differentiated iWAT adipocytes from the KO and WT mice (ESM Fig. 6a-c). Rosiglitazone is a PPAR $\gamma$ agonist that induces PPAR $\gamma$ expression and increases its activity [6, 38]. Consistently, rosiglitazone treatment significantly enhanced expression of Pparg, Cebpa, Fabp4, Adipoq and Ucp1 in brown adipocytes (Fig. 5d).

To confirm whether PPAR $\gamma$ mediates the effects of $m T O R$ deficiency on adipogenesis, we treated the WT and KO brown adipocytes with rosiglitazone. Remarkably, rosiglitazone treatment rescued the differentiation of the $m$ TOR-deficient brown pre-adipocytes (Fig. 5e). Consistently, the expression of Fabp4, Adipoq and Ucpl in brown adipocytes was significantly increased in both WT and KO brown adipocytes after rosiglitazone treatment (Fig. 5f). Taken together, we conclude that $m T O R$ deletion affects adipogenesis, at least in part, through the PPAR $\gamma$ signalling pathway.

\section{Discussion}

Here, we generated the Adipoq-mTOR mouse model and directly identified a role for $m T O R$ in BAT and WAT. We provided evidence that deletion of $m$ TOR inhibits fat development and leads to insulin resistance. Consistently, we demonstrated that $m T O R$ deletion decreases lipid accumulation and differentiation of brown and white adipocytes. We further elucidated the PPAR $\gamma$ signalling through which mTOR regulates the differentiation of pre-adipocytes. Our study reveals the critical role of the mTOR signalling pathway in regulating adipose tissues development, whole-body energy metabolism and insulin sensitivity.

The roles of $\mathrm{mTORC} 1$ and $\mathrm{mTORC} 2$ in adipose tissues have been studied separately by genetic perturbation of key components involved [15, 17, 24, 30], but the overall role of mTOR signalling has not been reported. Here, we used a highly adipocyte-specific Adipoq-Cre [31, 34, 35] to delete $m$ TOR in mice and demonstrated that $m T O R$ deletion inhibits BAT and WAT development. We found that adipose-specific deletion of mTOR dramatically decreased the levels of mTOR and phosphorylated S6 and 4EBP1 proteins but, surprisingly, increased phosphorylated AKT (S473) in BAT and iWAT. The increased levels of phosphorylated AKT might be due to the feedback activation of AKT signalling induced by $\mathrm{mTORC} 1$ deletion or a kinase that phosphorylates AKT, such as DNA-protein kinase 
a

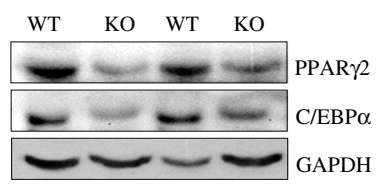

c

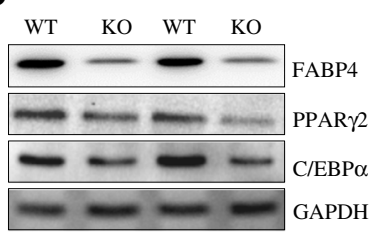

e

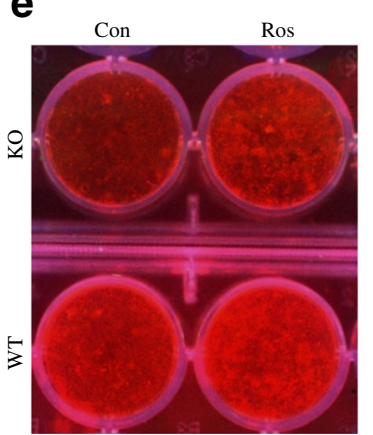

f b

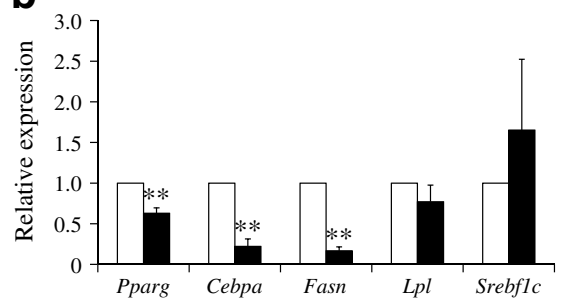

d

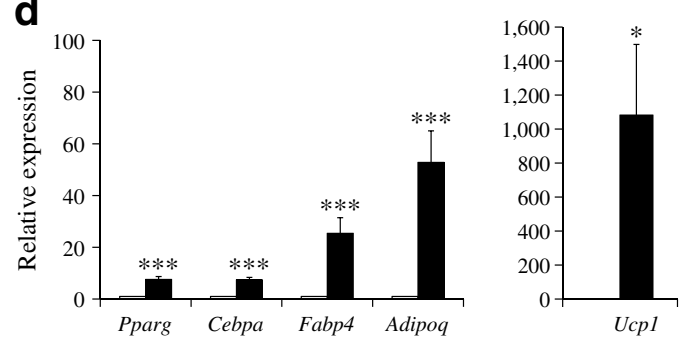

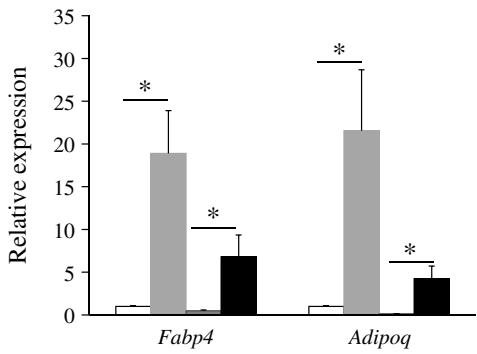

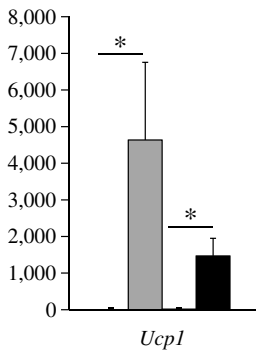

Fig. $5 m T O R$ deletion affects brown pre-adipocyte differentiation through PPAR $\gamma$. (a) Protein levels of PPAR $\gamma$ and C/EBP $\alpha$ in BAT of Adipoq-mTOR (KO) and WT mice. (b) mRNA levels of Pparg, Cebpa, Fasn, Lpl and Srebflc in BAT ( $n=4$ or 5). White bars, WT mice; black bars, KO mice. (c) Protein levels of FABP4, PPAR $\gamma$ and $\mathrm{C} / \mathrm{EBP} \alpha$ in brown adipocytes. (d) mRNA levels of Pparg, Cebpa, Fabp4, Adipoq and $U c p l$ in brown adipocytes $(n=6)$. White bars, vehicle control group; black bars, rosiglitazone (Ros) group. (e) Oil Red O staining of brown adipocytes treated with or without Ros. (f) mRNA levels of Fabp4, Adipoq and Ucpl in brown adipocytes treated with or without Ros $(n=6)$. White bars, WT cells treated with vehicle; light-grey bars, WT cells treated with Ros; dark-grey bars, $\mathrm{KO}$ cells treated with vehicle; black bars, KO cells treated with Ros. Error bars represent SEM; ${ }^{*} p<0.05$, ${ }^{* *} p<0.01$ and ${ }^{* * *} p<0.001$ for KO vs WT, Ros vs control or indicated comparisons. GAPDH, glyceraldehyde 3-phosphate dehydrogenase

in which the mass of Myf5-lineage adipocytes was decreased [15]. The different phenotypes from these studies may be due to the $\mathrm{Cre}$ mice that were used. Although BAT and some white adipocytes are from $M y f 5$ lineage, the $M y f 5$-Cre also drives gene deletion in skeletal muscles [42,43], which can secrete myokines that regulate adipose development and function [3, 33]. Moreover, leaky expression of FABP4-Cre was found in adipocyte progenitors and non-adipose tissues [31, 32, 44]. Therefore, the leaky expression of FABP4-Cre or Myf5-Cre makes it difficult to interpret the data from these studies. Our Adipoq-mTOR mouse model drives specific deletion of $m T O R$ in adipocytes and provides an excellent model with which to examine the role of mTOR in adipocytes and adipose tissues.

We found that adipocyte-specific deletion of $m T O R$ drives browning of WAT. The KO iWAT has numerous small adipocytes with higher levels of Ucp 1 and Lipe expression. Likewise, the browning of WAT is also commonly seen in lipodystrophic notypes observed in Myf5-Cre driven Rictor-knockout mice, 
models, such as Bscl2 knockout mice [45], in which fatty acids produced from unbridled lipolysis induce UCP1 and browning of WAT [46]. Moreover, previous studies have also reported that adipose-specific deletion of Raptor upregulated the expression of genes related to mitochondrial biogenesis in WAT [24]. $S 6 \mathrm{k} 1$ deletion also induces formation of multilocular adipocytes and increases expression of Ucp1 and Pgcla [25]. In contrast, deletion of $T s c 1$ induced the brown-to-white adipocyte phenotypic switch [26]. These results indicate that inhibition of mTORC1 signalling contributes to the white-to-brown adipocyte switching. In addition, a recent study reported that adipocytes lacking Rictor have stronger UCP1 staining and brownadipocyte-like characteristics [15], suggesting that mTORC2 may also regulate white-to-brown switching.

Adipose tissues participate in regulating whole-body energy metabolism, glucose homeostasis and insulin sensitivity [17]. Notably, we found that adipocyte-specific deletion of $m T O R$ has no effect on whole-body energy metabolism but causes insulin resistance. It has been reported that $\mathrm{mTORC} 1$ is a regulator of adipose metabolism and controls whole-body energy homeostasis [24]. Adipose-specific deletion of Raptor improves energy expenditure and insulin sensitivity and protects mice against HFD-induced obesity [24]. Consistently, S6kl-deficient mice have enhanced $\beta$-oxidation, faster glucose clearance and insulin sensitivity and are resistant to HFD-induced obesity [25]. However, administration of rapamycin prevents HFD-induced obesity and exacerbates glucose intolerance [41]. Distinct from mTORC1, loss of Rictor reduces glucose transport in fat cells $[16,17]$ and adipose-specific knockout of Rictor in mice leads to mild glucose intolerance and severe insulin resistance [17]. These reports suggest that the insulin resistance in Adipoq-mTOR mice might mainly result from the absence of mTOC2 in adipose tissues. However, we cannot exclude the effects of other factors, such as adipose tissue inflammation (data not shown), on insulin resistance in the KO mice.

Numerous studies have shown that hepatic steatosis is strongly associated with insulin resistance [47]. Notably, higher serum NEFA and increased hepatic steatosis were found in the Adipoq-mTOR mice. Consistent with our results, adipose-specific deletion of Rictor increases hepatic steatosis $[17,30]$. However, the weight of liver was not affected in the S6k1-deletion mice [25] and even less lipid accumulation was found after rapamycin treatment [41]. Taken together, the insulin resistance in Adipoq-mTOR mice may be mainly due to hepatic steatosis induced by $\mathrm{mTOC} 2$ deficiency.

We found that deletion of $m T O R$ inhibits pre-adipocyte differentiation through PPAR $\gamma$, a master regulator of adipogenesis [37]. Treatment with the PPAR agonist rosiglitazone rescued the differentiation and lipid accumulation in $m T O R$-knockout adipocytes. Previous studies have demonstrated that mTORC1 affects adipocyte differentiation and lipid accumulation by regulating lipogenesis and lipolysis [48]. In 3 T3-L1 adipocytes, activation of mTORC1 signalling suppresses lipolysis and promotes lipid accumulation [48]. Basal rates of lipolysis were increased in $S 6 \mathrm{kl}$-knockout mice [25]. Inhibition of mTORC1 signalling by knockdown of raptor or by rapamycin stimulates lipolysis by activation of Pnpla2 [48]. In addition, S6K1 depletion or rapamycin treatment inhibits adipocyte differentiation $[13,19,20]$. mTOR regulates pre-adipocyte differentiation and PPAR $\gamma$ activity probably not through the production of a ligand but rather through its direct impact on PPAR $\gamma$ transactivation activity [49] or through upregulation of AKT, a regulator of PPAR [12]. However, absence of Raptor in Myf5-lineage cells promotes osteogenesis and inhibits adipogenesis, while deletion of Rictor promotes adipogenesis and inhibits osteogenesis [50]. Therefore, mTORC1 and mTORC2 appear to counteract each other during adipocyte differentiation.

In conclusion, we demonstrate that mTOR plays several important roles in adipose development and adipocyte differentiation. These results provide a novel insight into the important role of mTOR in systemic metabolism. Such knowledge may be useful for developing strategies for treating obesity and related metabolic diseases.

Acknowledgements We thank J. Wu (Department of Animal Sciences, Purdue University, West Lafayette, IN, USA) for mouse colony maintenance and technical support, and members of the Kuang Laboratory for comments.

Funding The project was partially supported by funding from the National Institutes of Health (NIH, R01AR060652) and an incentive grant from Purdue University Office of Vice President for Research (OVPR) to SHK, 'Hundred Talents Program' funding from Zhejiang University to TZS and Nanhu Scholars Program for Young Scholars of Xinyang Normal University (XYNU) to PPZ.

Duality of interest The authors declare that there is no duality of interest associated with this manuscript.

Contribution statement TZS, PPZ and SHK designed and conceived the research. TZS, PPZ, QYJ and YX performed the experiments, analysed and interpreted the data and revised the manuscript. TZS and SHK wrote the manuscript. YZW assisted with data analysis and interpretation and with revision of the article. All authors approved the final version of the manuscript. TZS and SHK are responsible for the integrity of the work as a whole.

\section{References}

1. Cannon B, Nedergaard J (2004) Brown adipose tissue: function and physiological significance. Physiol Rev 84:277-359

2. Walden TB, Hansen IR, Timmons JA, Cannon B, Nedergaard J (2012) Recruited vs. nonrecruited molecular signatures of brown, brite, and white adipose tissues. Am J Physiol Endocrinol Metab 302:E19-E31

3. Bostrom P, Wu J, Jedrychowski MP et al (2012) A PGC1- $\alpha-$ dependent myokine that drives brown-fat-like development of white fat and thermogenesis. Nature 481:463-468 
4. Cao L, Choi EY, Liu XL et al (2011) White to brown fat phenotypic switch induced by genetic and environmental activation of a hypothalamic-adipocyte axis. Cell Metab 14:324-338

5. Fisher FM, Kleiner S, Douris N et al (2012) FGF21 regulates PGC$1 \alpha$ and browning of white adipose tissues in adaptive thermogenesis. Genes Dev 26:271-281

6. Ohno H, Shinoda K, Spiegelman BM, Kajimura S (2012) PPAR $\gamma$ agonists induce a white-to-brown fat conversion through stabilization of PRDM16 protein. Cell Metab 15:395-404

7. Bi P, Shan T, Liu W et al (2014) Inhibition of Notch signaling promotes browning of white adipose tissue and ameliorates obesity. Nat Med 20:911-918

8. Crane JD, Palanivel R, Mottillo EP et al (2015) Inhibiting peripheral serotonin synthesis reduces obesity and metabolic dysfunction by promoting brown adipose tissue thermogenesis. Nat Med 21: 166-172

9. Rosen ED, Spiegelman BM (2014) What we talk about when we talk about fat. Cell 156:20-44

10. Lamming DW, Sabatini DM (2013) A central role for mTOR in lipid homeostasis. Cell Metab 18:465-469

11. Laplante M, Sabatini DM (2012) mTOR signaling in growth control and disease. Cell 149:274-293

12. Yoon MS, Zhang C, Sun Y, Schoenherr CJ, Chen J (2013) Mechanistic target of rapamycin controls homeostasis of adipogenesis. J Lipid Res 54:2166-2173

13. Carnevalli LS, Masuda K, Frigerio F et al (2010) S6K1 plays a critical role in early adipocyte differentiation. Dev Cell 18:763-774

14. Soukas AA, Kane EA, Carr CE, Melo JA, Ruvkun G (2009) Rictor/ TORC2 regulates fat metabolism, feeding, growth, and life span in Caenorhabditis elegans. Genes Dev 23:496-511

15. Hung CM, Calejman CM, Sanchez-Gurmaches J et al (2014) Rictor/mTORC2 loss in the Myf5 lineage reprograms brown fat metabolism and protects mice against obesity and metabolic disease. Cell Rep 8:256-271

16. Olsen JM, Sato M, Dallner OS et al (2014) Glucose uptake in brown fat cells is dependent on mTOR complex 2-promoted GLUT1 translocation. J Cell Biol 207:365-374

17. Kumar A, Lawrence JC, Jung DY et al (2010) Fat cell-specific ablation of Rictor in mice impairs insulin-regulated fat cell and whole-body glucose and lipid metabolism. Diabetes 59:1397-1406

18. El-Chaar D, Gagnon A, Sorisky A (2004) Inhibition of insulin signaling and adipogenesis by rapamycin: effect on phosphorylation of p70 S6 kinase vs eIF4E-BP1. Int J Obes 28:191-198

19. Bell A, Grunder L, Sorisky A (2000) Rapamycin inhibits human adipocyte differentiation in primary culture. Obes Res 8:249-254

20. Yeh WC, Bierer BE, Mcknight SL (1995) Rapamycin inhibits clonal expansion and adipogenic differentiation of 3t3-L1 cells. Proc Natl Acad Sci U S A 92:11086-11090

21. Vila-Bedmar R, Lorenzo M, Fernandez-Veledo S (2010) Adenosine 5 '-monophosphate-activated protein kinase-mammalian target of rapamycin cross talk regulates brown adipocyte differentiation. Endocrinology 151:980-992

22. Chang GR, Chiu YS, Wu YY et al (2009) Rapamycin protects against high fat diet-induced obesity in $\mathrm{C} 57 \mathrm{BL} / 6 \mathrm{~J}$ mice. J Pharmacol Sci 109:496-503

23. Polak P, Hall MN (2009) mTOR and the control of whole body metabolism. Curr Opin Cell Biol 21:209-218

24. Polak P, Cybulski N, Feige JN, Auwerx J, Ruegg MA, Hall MN (2008) Adipose-specific knockout of raptor results in lean mice with enhanced mitochondrial respiration. Cell Metab 8:399-410

25. Um SH, Frigerio F, Watanabe M et al (2004) Absence of S6K1 protects against age- and diet-induced obesity while enhancing insulin sensitivity. Nature 431:200-205

26. Xiang X, Lan H, Tang H et al (2015) Tuberous sclerosis complex 1mechanistic target of rapamycin complex 1 signaling determines brown-to-white adipocyte phenotypic switch. Diabetes 64:519-528
27. Guertin DA, Stevens DM, Thoreen CC et al (2006) Ablation in mice of the mTORC components raptor, rictor, or mLST8 reveals that mTORC2 is required for signaling to Akt-FOXO and PKCalpha, but not S6K1. Dev Cell 11:859-871

28. Shiota C, Woo JT, Lindner J, Shelton KD, Magnuson MA (2006) Multiallelic disruption of the rictor gene in mice reveals that mTOR complex 2 is essential for fetal growth and viability. Dev Cell 11: 583-589

29. Jones KT, Greer ER, Pearce D, Ashrafi K (2009) Rictor/TORC2 regulates Caenorhabditis elegans fat storage, body size, and development through sgk-1. PLoS Biol 7:e60

30. Cybulski N, Polak P, Auwerx J, Ruegg MA, Hall MN (2009) mTOR complex 2 in adipose tissue negatively controls wholebody growth. Proc Natl Acad Sci U S A 106:9902-9907

31. Lee KY, Russell SJ, Ussar S et al (2013) Lessons on conditional gene targeting in mouse adipose tissue. Diabetes 62:864-874

32. Wang JQ, Liu RX, Wang F et al (2013) Ablation of LGR4 promotes energy expenditure by driving white-to-brown fat switch. Nat Cell Biol 15:1455-1463

33. Shan TZ, Liang XR, Bi PP, Kuang SH (2013) Myostatin knockout drives browning of white adipose tissue through activating the AMPK-PGC1 alpha-Fndc5 pathway in muscle. FASEB J 27: 1981-1989

34. Jeffery E, Berry R, Church CD et al (2014) Characterization of Cre recombinase models for the study of adipose tissue. Adipocytes 3 : 206-211

35. Wang ZV, Deng Y, Wang QA, Sun K, Scherer PE (2010) Identification and characterization of a promoter cassette conferring adipocyte-specific gene expression. Endocrinology 151:29332939

36. Stanford KI, Middelbeek RJW, Townsend KL et al (2013) Brown adipose tissue regulates glucose homeostasis and insulin sensitivity. J Clin Invest 123:215-223

37. Rosen ED, MacDougald OA (2006) Adipocyte differentiation from the inside out. Nat Rev Mol Cell Biol 7:885-896

38. Barquissau V, Beuzelin D, Giroud M et al (2014) PPAR gamma and PPAR alpha agonists induce white-to-brown conversion of human white adipocytes along with a metabolic shift from glucose to fatty acid oxidation. Diabetologia 57:S283

39. Davidson D, Amrein L, Panasci L, Aloyz R (2013) Small molecules, inhibitors of DNA-PK, targeting DNA repair, and beyond. Front Pharmacol 4:5

40. Zhu MY, Chen D, Li DY et al (2013) Luteolin inhibits angiotensin II-induced human umbilical vein endothelial cell proliferation and migration through downregulation of Src and Akt phosphorylation. Circ J 77:772-779

41. Chang GR, Wu YY, Chiu YS et al (2009) Long-term administration of rapamycin reduces adiposity, but impairs glucose tolerance in high-fat diet-fed KK/HIJ mice. Basic Clin Pharmacol 105:188-198

42. Kuang S, Kuroda K, Le Grand F, Rudnicki MA (2007) Asymmetric self-renewal and commitment of satellite stem cells in muscle. Cell 129:999-1010

43. Dong F, Sun X, Liu W et al (2006) Pitx2 promotes development of splanchnic mesoderm-derived branchiomeric muscle. Development 133:4891-4899

44. Shan TZ, Liu WY, Kuang SH (2013) Fatty acid binding protein 4 expression marks a population of adipocyte progenitors in white and brown adipose tissues. FASEB J 27:277-287

45. Chen W, Zhou H, Liu S et al (2013) Altered lipid metabolism in residual white adipose tissues of Bscl2 deficient mice. Plos One 8: e82526

46. Fedorenko A, Lishko PV, Kirichok Y (2012) Mechanism of fattyacid-dependent UCP1 uncoupling in brown fat mitochondria. Cell 151:400-413 
47. Monetti M, Levin MC, Watt MJ et al (2007) Dissociation of hepatic steatosis and insulin resistance in mice overexpressing DGAT in the liver. Cell Metab 6:69-78

48. Chakrabarti P, English T, Shi J, Smas CM, Kandror KV (2010) Mammalian target of rapamycin complex 1 suppresses lipolysis, stimulates lipogenesis, and promotes fat storage. Diabetes 59: 775-781
49. Kim JE, Chen J (2004) regulation of peroxisome proliferatoractivated receptor-gamma activity by mammalian target of rapamycin and amino acids in adipogenesis. Diabetes 53:27482756

50. Martin SK, Fitter S, Dutta AK et al (2015) Brief report: the differential roles of mTORC1 and mTORC2 in mesenchymal stem cell differentiation. Stem Cells 33:1359-1365 